\title{
Thioacetalization of the carbonyl function, transthioacetalization of acetals, ketals, oximes and hydrazones catalysed by aqueous hydrobromic acid
}

\author{
Popat D. Shinde, Hanumant B. Borate, and Radhika D. Wakharkar* \\ Division of Organic Chemistry: Technology, National Chemical Laboratory, \\ Pune 411 008, India \\ E-mail:rdw@dalton.ncl.res.in
}

(received 28 Jul 04; accepted 23 Sept 04; published on the web 28 Sept 04)

\begin{abstract}
Thioacetalization of a variety of aldehydes and ketones was achieved in excellent yields at room temperature using thiols or dithiols in aqueous hydrobromic acid. Thioacetals were also prepared by transthioacetalization of oximes, hydrazones, acetals and ketals under similar conditions. The use of aqueous hydrochloric acid, hydrofluoric acid and hydriodic acid for this transformation was also explored.
\end{abstract}

Keywords: Thioacetalization, transthioacetalization, acetals, ketals, oximes, hydrazones, hydrobromic acid

\section{Introduction}

The protection of the carbonyl group as thioacetal is often a necessary step in the synthesis of complex organic molecules due to its inbuilt stability for acidic or basic conditions. Thioacetals are also useful in organic synthesis as acyl carbanion equivalents in $\mathrm{C}-\mathrm{C}$ bond forming reactions. ${ }^{1}$ Preparation of thioacetals ${ }^{2}$ generally involves reaction of carbonyl compounds with thiols in presence of protic or Lewis acids in various dry organic solvents many of which are environmentally hazardous.

Though there are a number of reagents reported for preparation of thioacetals, the continuous search for novel reagents with selectivity, ease of operation or increase in yields has resulted in several publications in recent years. ${ }^{2 g-o}$ Water is considered as a favorable solvent in many organic transformations ${ }^{3}$ and thioacetalization in aqueous medium has been reported recently using indium tribromide ${ }^{4}$ and dodecylbenzenesulfonic acid ${ }^{5}$ but the possibility of preparing thioacetals using aqueous halo acids (hydrobromic, hydrochloric and hydrofluoric) has not been explored. We ventured to explore the possibility of preparing thioacetals in aqueous medium 
using various halo acids as catalyst and wish to report herein our results demonstrating use of catalytic amount of hydrobromic acid to prepare thioacetals from aldehydes and ketones as well as for transthioacetalization of acetals, ketals, oximes and hydrazones as shown in Scheme 1.

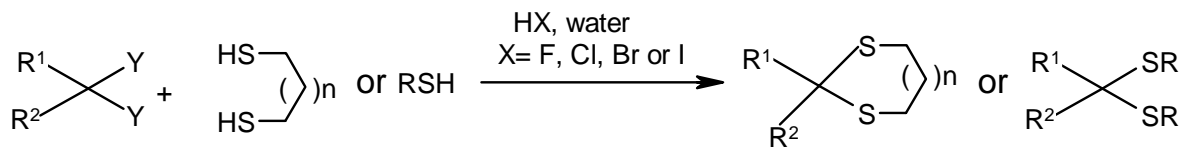

\section{Scheme 1}

\section{Results and Discussion}

Reaction of 3,4,5-trimethoxybenzaldehyde with ethanedithiol in the presence of commercially available aqueous hydrobromic acid $(48 \% \mathrm{w} / \mathrm{w}$ aq.) at room temperature afforded the corresponding thioacetal in high yield. The generality of this transformation was confirmed by reacting various carbonyl compounds with dithiols under similar conditions. Table 1 shows the results of the thioacetalization of various carbonyl compounds including variably substituted aldehydes. This protocol was also effective for ketones as exemplified by entries 13 and 14 in Table 1.

Table 1. Thioacetalization of aldehydes and ketones in the presence of hydrobromic acid $(0.2$ eq., $48 \%$ w/w aq.)

\begin{tabular}{ccccc}
\hline Entry & Substrate & $\mathrm{n}$ & Time (min.) & Yield \% \\
\hline 1 & 3,4,5-Trimethoxybenzaldehyde & 0 & 15 & 94 \\
2 & 3,4,5-Trimethoxybenzaldehyde & 1 & 15 & 92 \\
3 & n-Butyraldehyde & 0 & 60 & 85 \\
4 & 3-Nitrobenzaldehyde & 0 & 60 & 94 \\
5 & 2,3-Dichlorobenzaldehyde & 0 & 90 & 92 \\
6 & 3-Nitrobenzaldehyde & 1 & 60 & 90 \\
7 & 4-Methoxybenzaldehyde & 0 & 20 & 93 \\
8 & Benzaldehyde & 0 & 15 & 96 \\
9 & 2-Furaldehyde & 0 & 15 & 92 \\
10 & 1-Naphthaldehyde & 0 & 60 & 81 \\
11 & Cinnamaldehyde & 0 & 60 & 89 \\
12 & Syringaldehyde & 0 & 120 & 74 \\
13 & Ethyl levulinate & 0 & 60 & 94 \\
14 & Cyclohexanone & 0 & 90 & 90 \\
15 & Chromone-3-carboxaldehyde & 0 & 60 & 87 \\
\hline
\end{tabular}


The desired products were obtained in high isolated yields. Similarly 3,4,5trimethoxybenzaldehyde when treated with ethanethiol under same conditions resulted in the corresponding thioacetal in high yield. It was noted that when syringaldehyde (entry 12, Table 1) was subjected to protection with ethanedithiol the complete conversion took longer time and with slight reduction in the yield which may be as a result of deactivation of carboxaldehyde due to the free hydroxyl in the para position.

In order to examine the utility of other halo acids in thioacetalization, 3,4,5trimethoxybenzaldehyde was reacted with ethanedithiol in presence of commercially available aqueous hydrochloric acid (36\% w/w aq., 0.2 eq.), or hydrofluoric acid (40\% w/w aq., 0.2 eq.) or hydriodic acid (57\% w/w aq., 0.2 eq.) under identical conditions and it was observed that the corresponding thioacetal was obtained in comparable yield from all the experiments.

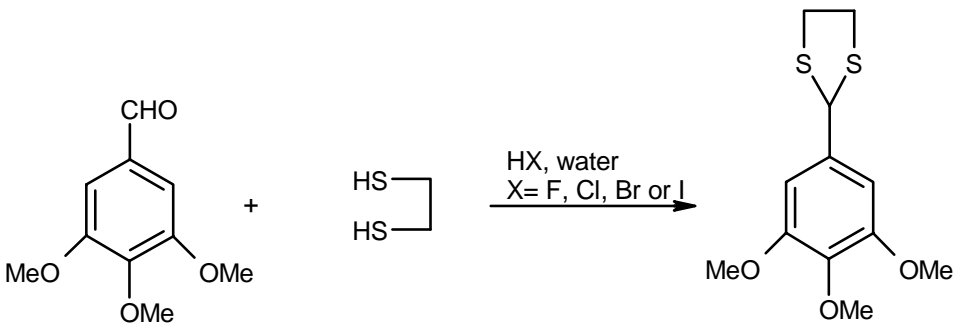

\section{Scheme 2}

Due to the stability of thioacetals, they have been of great interest to organic chemists and there are a number of reports of transthioacetalization of acetals, ketals or O, S-acetals ${ }^{6}$, oximes ${ }^{7}$ and hydrazones ${ }^{8}$. The results obtained in case of thioacetalization prompted us to study the scope of the utility of halo acids for transthioacetalization. Accordingly, acetals, ketals, oximes and hydrazones were reacted with dithiols in presence of aqueous hydrobromic acid (48\% $\mathrm{w} / \mathrm{w})$ (Scheme 3) and it was found that the thioacetals were obtained in high isolated yields (Table 2).

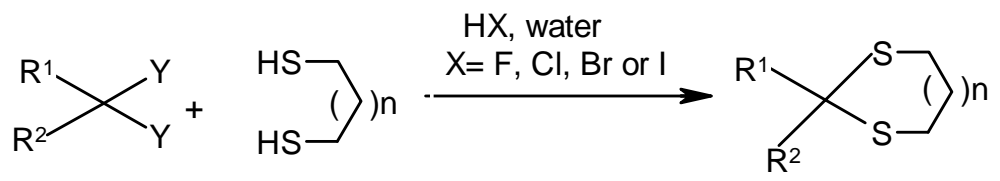

\section{Scheme 3}

Transthioacetalization of acetals and ketals was achieved at room temperature while oximes and hydrazones required refluxing temperature and somewhat longer reaction times. It is evident from Table 2 that the transthioacetalization of oximes and hydrazones using aqueous hydrobromic acid (48\% w/w aq.) can be achieved successfully irrespective of the electronic nature of functional groups. 
Table 2. Transthioacetalization of oximes, hydrazones and ketals in the presence of hydrobromic acid $(0.2$ eq., $48 \%$ w/w aq.)

\begin{tabular}{|c|c|c|c|c|c|}
\hline Entry & Substrate & $\mathrm{Y}, \mathrm{Y}$ & $\mathrm{n}$ & $\begin{array}{c}\text { Time } \\
\text { (hr) }\end{array}$ & Yield \% \\
\hline 1 & 3,4,5-Trimethoxybenzaldehyde & $=\mathrm{N}-\mathrm{OH}$ & 0 & 2 & 92 \\
\hline 2 & 2,3-Dichlorobenzaldehyde & $=\mathrm{N}-\mathrm{OH}$ & 0 & 2 & 88 \\
\hline 3 & Cyclohexanone & $=\mathrm{N}-\mathrm{OH}$ & 0 & 4 & 90 \\
\hline 4 & 3-Nitrobenzaldehyde & $=\mathrm{N}-\mathrm{OH}$ & 0 & 2 & 90 \\
\hline 5 & 3-Nitrobenzaldehyde & $=\mathrm{N}-\mathrm{OH}$ & 1 & 6 & 86 \\
\hline 6 & 2-Nitrobenzaldehyde & $=\mathrm{NNHPh}$ & 0 & 6 & 90 \\
\hline 7 & 3-Nitrobenzaldehyde & $=\mathrm{NNHPh}$ & 0 & 4 & 92 \\
\hline 8 & 4-Methoxybenzaldehyde & $=\mathrm{NNHPh}$ & 0 & 4 & 76 \\
\hline 9 & 2-Furaldehyde & $=\mathrm{NNHPh}$ & 0 & 2 & 94 \\
\hline 10 & 4-Hydroxybenzaldehyde & $=\mathrm{NNHPh}$ & 0 & 2 & 90 \\
\hline 11 & 2,3-Dichlorobenzaldehyde & $=\mathrm{NNHPh}$ & 0 & 8 & 84 \\
\hline 12 & 3,4,5-Trimethoxybenzaldehyde & $=\mathrm{NNHPh}$ & 0 & 2 & 94 \\
\hline 13 & n-Butyraldehyde & $=\mathrm{NNHPh}$ & 0 & 0.25 & 94 \\
\hline 14 & 2-Acetylfuran & $-\mathrm{OCH}_{2} \mathrm{CH}_{2} \mathrm{O}-$ & 0 & 1 & 89 \\
\hline 15 & 3,4, 5-Trimethoxybenzaldehyde & $-\mathrm{OCH}_{2} \mathrm{CH}_{2} \mathrm{O}-$ & 0 & 0.25 & 94 \\
\hline 16 & Cyclohexanone & $-\mathrm{OCH}_{2} \mathrm{CH}_{2} \mathrm{O}-$ & 0 & 2 & 83 \\
\hline
\end{tabular}

\section{Experimental Section}

General Procedures. Melting points are uncorrected. ${ }^{1} \mathrm{H}$ NMR and ${ }^{13} \mathrm{C}$ NMR spectra were recorded on a Bruker Avance DPX 200 (200 and 50.3 MHz, respectively) spectrometer in organic solvents with TMS as internal standard. IR spectra were recorded on Perkin-Elmer Spectrum 683 B or 160S FT IR spectrophotometer. Elemental analysis was performed on a Perkin-Elmer Model 2400 analyser. Thin layer chromatography was performed on silica/alumina plates. Column chromatography was performed on silica gel (60-120 mesh). Preparation of oximes, phenylhydrazones and oxyacetals were carried out according to the general procedure. ${ }^{9}$

\section{Typical experimental procedure for thioacetalization}

To a mixture of 3,4,5-trimethoxybenzaldehyde $(0.50 \mathrm{~g}, 2.55 \mathrm{mmol})$ and 1,2- ethanedithiol $(0.29 \mathrm{~g}, 3.06 \mathrm{mmol})$ in water $(5 \mathrm{ml})$, was added aqueous hydrobromic acid $(48 \% \mathrm{w} / \mathrm{w}, 0.06 \mathrm{ml}$, $0.51 \mathrm{mmol}$ ). The reaction mixture was stirred at room temperature for $15 \mathrm{~min}$ and the reaction was monitored by thin layer chromatography (Table 1, entry 1 ). The reaction mixture was then extracted with chloroform $(2 \times 15 \mathrm{ml})$, combined organic layer was washed with $10 \%$ sodium hydroxide solution $(10 \mathrm{ml})$ followed by water $(10 \mathrm{ml})$ and brine $(5 \mathrm{ml})$. Concentration of organic 
layer under reduced pressure and purification of residue by passing through silica gel furnished 2-(3,4,5-trimethoxyphenyl)-1,3-dithiolane (0.69 g, $94 \%)$ as a pale yellow solid.

\section{Typical procedure for transthioacetalization}

To a mixture of 3,4,5-trimethoxybenzaldoxime $(0.40 \mathrm{~g}, 1.88 \mathrm{mmol})$ and 1,2- ethanedithiol $(0.21 \mathrm{~g}, 2.25 \mathrm{mmol})$, in water $(5 \mathrm{ml})$, was added aqueous hydrobromic acid (48 \% w/w, $0.05 \mathrm{ml}$, $0.41 \mathrm{mmol}$ ). The reaction mixture was refluxed for $2 \mathrm{~h}$ (table 2, entry 1 ). It was then cooled to room temperature and extracted with chloroform $(2 \times 15 \mathrm{ml})$. The combined organic layer was washed with $10 \%$ sodium hydroxide solution $(10 \mathrm{ml})$ followed by water $(10 \mathrm{ml})$ and brine $(5 \mathrm{ml})$. Concentration of organic layer under reduced pressure and purification of residue by passing through silica gel furnished 2-(3,4,5-trimethoxyphenyl)-1,3-dithiolane $(0.45 \mathrm{~g}, 92 \%)$ as a pale yellow solid.

M. p. 53-54 ${ }^{\circ} \mathrm{C} ;{ }^{1} \mathrm{H}$ NMR $\left(200 \mathrm{MHz}, \mathrm{CDCl}_{3}+\mathrm{CCl}_{4}\right): \delta 3.22-3.47(\mathrm{~m}, 4 \mathrm{H}), 3.77(\mathrm{~s}, 3 \mathrm{H}), 3.81(\mathrm{~s}$, $6 \mathrm{H}), 5.53(\mathrm{~s}, 1 \mathrm{H}), 6.72(\mathrm{~s}, 2 \mathrm{H}) ;{ }^{13} \mathrm{C} \mathrm{NMR}\left(50.3 \mathrm{MHz}, \mathrm{CDCl}_{3}+\mathrm{CCl}_{4}\right): \delta 39.81(2 \mathrm{C}), 55.80(2 \mathrm{C})$, 56.61, 60.40, 104.87 (2C), 134.98, 137.59, 152.81 (2C); Mass (m/z): $272\left(\mathrm{M}^{+}\right)$. Anal. Calcd. for $\mathrm{C}_{12} \mathrm{H}_{16} \mathrm{O}_{3} \mathrm{~S}_{2}$ : C 52.91; H 5.92; S 23.54. Found C 52.98; H 6.02; S 23.63\%.

2-(3,4,5-Trimethoxyphenyl)-1,3-dithiane. ${ }^{7}$ White solid; $\mathrm{mp} 85-86^{\circ} \mathrm{C}$; IR (v max, $\left.\mathrm{CHCl}_{3}\right): 760$ and $1600 \mathrm{~cm}^{-1} ;{ }^{1} \mathrm{H}$ NMR $\left(200 \mathrm{MHz}, \mathrm{CDCl}_{3}+\mathrm{CCl}_{4}\right): \delta 1.75-2.30(\mathrm{~m}, 2 \mathrm{H}), 2.80-3.15(\mathrm{~m}, 4 \mathrm{H}), 3.81$ $(\mathrm{m}, 3 \mathrm{H}), 3.86(\mathrm{~s}, 6 \mathrm{H}), 5.06(\mathrm{~s}, 1 \mathrm{H}), 6.67(\mathrm{~s}, 2 \mathrm{H}) ;{ }^{13} \mathrm{C} \mathrm{NMR}\left(50.3 \mathrm{MHz}, \mathrm{CDCl}_{3}\right): \delta 25.04,32.20$ (2C), 51.87, 56.10 (2C), 60.77, 104.73 (2C), 134.80, 137.81, 153.29 (2C); Mass (m/z): $286\left(\mathrm{M}^{+}\right)$. Anal. Calcd. for $\mathrm{C}_{13} \mathrm{H}_{18} \mathrm{O}_{3} \mathrm{~S}_{2}$ : C 54.52; H 6.33; S 22.39. Found C 54.64; H 6.43; S 22.52\%.

2-Propyl-1,3-dithiolane. Colorless liquid; ${ }^{1} \mathrm{H}$ NMR $\left(200 \mathrm{MHz}, \mathrm{CDCl}_{3}+\mathrm{CCl}_{4}\right): \delta 0.93(\mathrm{t}, 3 \mathrm{H})$, 1.35-1.55 (m, 2H), 1.70-1.90 (m, 2H), 3.00-3.45 (m, 4H), $4.46(\mathrm{t}, J=7 \mathrm{~Hz}, 1 \mathrm{H}) ;{ }^{13} \mathrm{C}$ NMR $(50.3$ $\left.\mathrm{MHz}, \mathrm{CDCl}_{3}+\mathrm{CCl}_{4}\right): \delta 13.45,22.19,38.00$ (2C), 41.20, 53.14; Mass (m/z): $147\left(\mathrm{M}^{+}\right)$. Anal. Calcd. for $\mathrm{C}_{6} \mathrm{H}_{12} \mathrm{~S}_{2}$ : C 48.60; $\mathrm{H}$ 8.16; S 43.25. Found C 48.67; H 8.25; S 43.37\%.

2-(3-Nitrophenyl)-1,3-dithiolane. Pale yellow solid; mp $62^{\circ} \mathrm{C}$; IR (v max, $\left.\mathrm{CHCl}_{3}\right)$ : 1215, 1352, 1532 and $3020 \mathrm{~cm}^{-1}$; ${ }^{1} \mathrm{H}$ NMR $\left(200 \mathrm{MHz}, \mathrm{CDCl}_{3}+\mathrm{CCl}_{4}\right): \delta 3.30-3.60(\mathrm{~m}, 4 \mathrm{H}), 5.63(\mathrm{~s}, 1 \mathrm{H}), 7.47$ (t, $J=8 \mathrm{~Hz}, 1 \mathrm{H}), 7.81(\mathrm{~d}, J=8 \mathrm{~Hz}, 1 \mathrm{H}), 8.09$ (dd, $J=8 \mathrm{~Hz}$ and $2 \mathrm{~Hz}, 1 \mathrm{H}), 8.37(\mathrm{~s}, 1 \mathrm{H}) ;{ }^{13} \mathrm{C} \mathrm{NMR}$ $\left(50.3 \mathrm{MHz}, \mathrm{CDCl}_{3}+\mathrm{CCl}_{4}\right): \delta 40.18$ (2C), 54.66, 122.70 (2C), 129.06, 133.80, 143.25, 147.95; Mass (m/z): $227\left(\mathrm{M}^{+}\right)$. Anal. Calcd. for $\mathrm{C}_{9} \mathrm{H}_{9} \mathrm{NO}_{2} \mathrm{~S}_{2}$ : C 47.56; H 3.99; N 6.16; S 28.21. Found C 47.68; H 4.06; N 6.31; S 28.34\%.

2-(2,3-Dichlorophenyl)-1,3-dithiolane. White solid; $\mathrm{mp} 58-59{ }^{\circ} \mathrm{C} ;{ }^{1} \mathrm{H} \mathrm{NMR}(200 \mathrm{MHz}$, $\left.\mathrm{CDCl}_{3}+\mathrm{CCl}_{4}\right): \delta 3.35-3.45(\mathrm{~m}, 4 \mathrm{H}), 6.07(\mathrm{~s}, 1 \mathrm{H}), 7.21(\mathrm{t}, J=8 \mathrm{~Hz}, 1 \mathrm{H}), 7.38(\mathrm{dd}, J=8 \mathrm{~Hz}$ and 2 $\mathrm{Hz}, 1 \mathrm{H}), 7.78(\mathrm{dd}, J=8 \mathrm{~Hz}$ and $2 \mathrm{~Hz}, 1 \mathrm{H}) ;{ }^{13} \mathrm{C} \mathrm{NMR}\left(50.3 \mathrm{MHz}, \mathrm{CDCl}_{3}+\mathrm{CCl}_{4}\right): \delta 38.00(2 \mathrm{C})$, 54.03, 127.90, 128.56, 128.90, 134.50, 134.89, 141.00; Mass (m/z): $250\left(\mathrm{M}^{+}\right)$. Anal. Calcd. for $\mathrm{C}_{9} \mathrm{H}_{8} \mathrm{Cl}_{2} \mathrm{~S}_{2}$ : C 43.03; $\mathrm{H} 3.21 ; \mathrm{Cl} 28.23 ; \mathrm{S}$ 25.53. Found C 43.07; H 3.28; $\mathrm{Cl} 28.32 ; \mathrm{S} \mathrm{25.57 \% .}$

2-(3-Nitrophenyl)-1,3-dithiane. Yellowish solid; mp 110-111 ${ }^{\circ} \mathrm{C} ;{ }^{1} \mathrm{H}$ NMR $(200 \mathrm{MHz}$, $\left.\mathrm{CDCl}_{3}+\mathrm{CCl}_{4}\right): \delta 1.75-2.05(\mathrm{~m}, 1 \mathrm{H}), 2.10-2.25(\mathrm{~m}, 1 \mathrm{H}), 2.80-3.15(\mathrm{~m}, 4 \mathrm{H}) 5.20(\mathrm{~s}, 1 \mathrm{H}), 7.49(\mathrm{t}$, $J=8 \mathrm{~Hz}, 1 \mathrm{H}), 7.77(\mathrm{~d}, J=8 \mathrm{~Hz}, 1 \mathrm{H}), 8.13(\mathrm{~d}, J=8 \mathrm{~Hz}, 1 \mathrm{H}), 8.31(\mathrm{~s}, 1 \mathrm{H}) ;{ }^{13} \mathrm{C} \mathrm{NMR}(50.3 \mathrm{MHz}$, $\left.\mathrm{CDCl}_{3}+\mathrm{CCl}_{4}\right): \delta 24.90,31.85(2 \mathrm{C}), 50.30,123.23,123.45,129.85,134.18,141.39$. 148.52; Mass 
$(\mathrm{m} / \mathrm{z}): 241\left(\mathrm{M}^{+}\right)$. Anal. Calcd. for $\mathrm{C}_{10} \mathrm{H}_{11} \mathrm{NO}_{2} \mathrm{~S}_{2}$ : C 49.77; H 4.59; N 5.80; $\mathrm{S}$ 26.57. Found $\mathrm{C}$ 49.85; H 4.72; N 5.88; S 26.64\%.

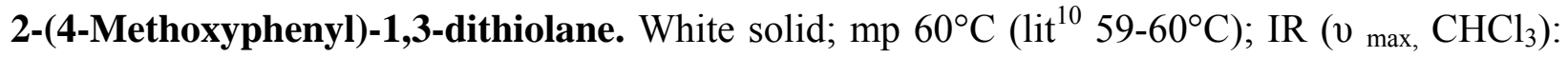
$1050,1250,1500,1600,2895$ and $2925 \mathrm{~cm}^{-1} ;{ }^{1} \mathrm{H}$ NMR $\left(200 \mathrm{MHz}, \mathrm{CDCl}_{3}+\mathrm{CCl}_{4}\right): \delta 3.25-3.55(\mathrm{~m}$, 4H), $3.79(\mathrm{~s}, 3 \mathrm{H}), 5.65(\mathrm{~s}, 1 \mathrm{H}), 6.85(\mathrm{~d}, J=10 \mathrm{~Hz}, 2 \mathrm{H}), 7.47(\mathrm{~d}, J=10 \mathrm{~Hz}, 2 \mathrm{H}) ;{ }^{13} \mathrm{C}$ NMR $(50.3$ $\left.\mathrm{MHz}, \mathrm{CDCl}_{3}+\mathrm{CCl}_{4}\right): \delta 39.96(2 \mathrm{C}), 55.03,55.91,113.62(2 \mathrm{C}), 128.99$ (2C), 131.56, 159.20; Mass $(\mathrm{m} / \mathrm{z}): 212\left(\mathrm{M}^{+}\right)$. Anal. Calcd. for $\mathrm{C}_{10} \mathrm{H}_{12} \mathrm{OS}_{2}$ : C 56.57; H 5.70; S 30.20. Found C 56.69; H 5.78; S $30.23 \%$.

2-Phenyl-1,3-dithiolane. ${ }^{20,10}$ Colorless thick oil; ${ }^{1} \mathrm{H}$ NMR (200 MHz, $\left.\mathrm{CDCl}_{3}+\mathrm{CCl}_{4}\right): \delta 3.19-3.50$ (m, 4H), $5.59(\mathrm{~s}, 1 \mathrm{H}), 3.81(\mathrm{~s}, 6 \mathrm{H}), 7.18-7.32(\mathrm{~m}, 3 \mathrm{H}), 7.45-7.55(\mathrm{~m}, 2 \mathrm{H}) ;{ }^{13} \mathrm{C} \mathrm{NMR}(50.3 \mathrm{MHz}$, $\left.\mathrm{CDCl}_{3}+\mathrm{CCl}_{4}\right): \delta 40.49(2 \mathrm{C}), 56.59,128.27(4 \mathrm{C}), 128.71,140.62$; Mass $(\mathrm{m} / \mathrm{z}): 182\left(\mathrm{M}^{+}\right)$. Anal. Calcd. for $\mathrm{C}_{9} \mathrm{H}_{10} \mathrm{~S}_{2}$ : C 59.29; H 5.53; S 35.18. Found C 59.42; H 5.61; $\mathrm{S} \mathrm{35.26 \% .}$

2-(1,3-Dithiolan-2-yl)-furan. ${ }^{11,12}$ Thick oil; IR (v max, neat): 1149, 1498, 1650 and $2900 \mathrm{~cm}^{-1}$; ${ }^{1} \mathrm{H}$ NMR $\left(200 \mathrm{MHz}, \mathrm{CDCl}_{3}+\mathrm{CCl}_{4}\right): \delta 3.25-3.50(\mathrm{~m}, 4 \mathrm{H}), 5.61(\mathrm{~s}, 1 \mathrm{H}), 6.28(\mathrm{~d}, J=2 \mathrm{~Hz}, 2 \mathrm{H})$, $7.36(\mathrm{dd}, J=6 \mathrm{~Hz}$ and $2 \mathrm{~Hz}, 1 \mathrm{H}), 8.24(\mathrm{~d}, J=6 \mathrm{~Hz}, 1 \mathrm{H}), 8.32(\mathrm{~s}, 1 \mathrm{H}) ;{ }^{13} \mathrm{C}$ NMR $(50.3 \mathrm{MHz}$, $\left.\mathrm{CDCl}_{3}+\mathrm{CCl}_{4}\right): \delta 39.28(2 \mathrm{C}), 47.66,107.20,110.55,142.68,154.55$; Mass $(\mathrm{m} / \mathrm{z}): 172\left(\mathrm{M}^{+}\right)$. Anal. Calcd. for $\mathrm{C}_{7} \mathrm{H}_{8} \mathrm{OS}_{2}$ : C 48.80; H 4.68; S 37.23. Found C 48.89; H 4.77; S 37.34\%.

2-Napthalen-1-yl-1,3-dithiolane. ${ }^{20}$ Thick colorless liquid; ${ }^{1} \mathrm{H}$ NMR (200 MHz, $\left.\mathrm{CDCl}_{3}+\mathrm{CCl}_{4}\right)$ : $\delta$ 3.30-3.60 (m, 4H), $6.54(\mathrm{~s}, 1 \mathrm{H}), 7.42-7.70(\mathrm{~m}, 3 \mathrm{H}), 7.75-7.90(\mathrm{~m}, 2 \mathrm{H}), 8.08(\mathrm{~d}, J=8 \mathrm{~Hz}, 1 \mathrm{H})$, $8.22(\mathrm{~d}, J=8 \mathrm{~Hz}, 1 \mathrm{H}) ;{ }^{13} \mathrm{C} \mathrm{NMR}\left(50.3 \mathrm{MHz}, \mathrm{CDCl}_{3}+\mathrm{CCl}_{4}\right): \delta 39.46(2 \mathrm{C}), 52.73,123.19,124.66$, 125.22, 125.62, 126.17, 128.71, 130.99, 133.71, 135.54; Mass (m/z): $232\left(\mathrm{M}^{+}\right)$. Anal. Calcd. for $\mathrm{C}_{13} \mathrm{H}_{12} \mathrm{~S}_{2}$ : C 67.20; H 5.21; S 27.60. Found C 67.34; H 5.31; S 27.67\%.

2-Styryl-1,3-dithiolane. Pale yellow solid; mp 56-58 ${ }^{\circ} \mathrm{C}$ (lit. ${ }^{10}$ 58-59; IR ( $v_{\max } \mathrm{CHCl}_{3}$ ): 760 and $2560 \mathrm{~cm}^{-1} ;{ }^{1} \mathrm{H}$ NMR $\left(200 \mathrm{MHz}, \mathrm{CDCl}_{3}+\mathrm{CCl}_{4}\right): \delta 3.20-3.50(\mathrm{~m}, 4 \mathrm{H}), 5.24(\mathrm{~d}, J=8 \mathrm{~Hz}, 1 \mathrm{H}), 6.24$ (dd, $J=16 \mathrm{~Hz}$ and $8 \mathrm{~Hz}, 1 \mathrm{H}), 6.51(\mathrm{~d}, J=14 \mathrm{~Hz}, 1 \mathrm{H}), 7.25-7.55(\mathrm{~m}, 5 \mathrm{H}) ;{ }^{13} \mathrm{C} \mathrm{NMR}(50.3 \mathrm{MHz}$, $\left.\mathrm{CDCl}_{3}+\mathrm{CCl}_{4}\right): \delta 39.86(2 \mathrm{C}), 54.75,126.91(2 \mathrm{C}), 128.05,128.78(2 \mathrm{C}), 129.41,130.33,136.32$; Mass (m/z): $208\left(\mathrm{M}^{+}\right)$. Anal. Calcd. for $\mathrm{C}_{11} \mathrm{H}_{12} \mathrm{~S}_{2}: \mathrm{C}$ 63.41; H 5.81; S 30.78. Found C 63.57; H 5.89 ; S $30.87 \%$.

4-(1,3-Dithiolan-2-yl)-2, 6-dimethoxylphenol. White solid; mp $65^{\circ} \mathrm{C}$; IR ( $\left.v_{\max } \mathrm{CHCl}_{3}\right): 1215$, 1515, 1615 and $3533 \mathrm{~cm}^{-1} ;{ }^{1} \mathrm{H}$ NMR $\left(200 \mathrm{MHz}, \mathrm{CDCl}_{3}+\mathrm{CCl}_{4}\right): \delta 3.21-3.49(\mathrm{~m}, 4 \mathrm{H}), 3.82(\mathrm{~s}$, $6 \mathrm{H}), 5.54(\mathrm{~s}, 1 \mathrm{H}), 6.73(\mathrm{~s}, 2 \mathrm{H}) ;{ }^{13} \mathrm{C} \mathrm{NMR}\left(50.3 \mathrm{MHz}, \mathrm{CDCl}_{3}+\mathrm{CCl}_{4}\right): \delta 40.23(2 \mathrm{C}), 56.41(2 \mathrm{C})$, 57.29, $104.96(2 \mathrm{C}), 130.40,134.77,146.94(2 \mathrm{C})$; Mass $(\mathrm{m} / \mathrm{z}): 257\left(\mathrm{M}^{+}\right)$. Anal. Calcd. for $\mathrm{C}_{11} \mathrm{H}_{14} \mathrm{O}_{3} \mathrm{~S}_{2}$ : C 51.14; H 5.46; S 24.82. Found C 51.18; H 5.52; $\mathrm{S} 24.90 \%$.

Ethyl 3-(2-methyl-1,3-dithiolan-2-yl)-propionate. Colorless liquid; ${ }^{1} \mathrm{H}$ NMR (200 MHz, $\left.\mathrm{CDCl}_{3}+\mathrm{CCl}_{4}\right): \delta 1.20(\mathrm{t}, J=8 \mathrm{~Hz}, 3 \mathrm{H}), 1.72(\mathrm{~s}, 3 \mathrm{H}), 2.12-2.25(\mathrm{~m}, 2 \mathrm{H}), 2.45-2.60(\mathrm{~m}, 2 \mathrm{H}), 3.27$ (s, $4 \mathrm{H}), 4.07$ (q, $J=8 \mathrm{~Hz}, 2 \mathrm{H}) ;{ }^{13} \mathrm{C}$ NMR $\left(50.3 \mathrm{MHz}, \mathrm{CDCl}_{3}+\mathrm{CCl}_{4}\right): \delta 13.85,31.60,32.38,39.32$, $39.84(2 \mathrm{C}), 59.87,65.53,172.50$; Mass (m/z): $119\left(\mathrm{M}^{+}\right)$. Anal. Calcd. for $\mathrm{C}_{9} \mathrm{H}_{16} \mathrm{O}_{2} \mathrm{~S}_{2}: \mathrm{C} 49.06 ; \mathrm{H}$ 7.32; S 29.10. Found C 49.19; H 7.38; S 29.17\%.

1, 4-Dithia-spiro-4,5-decane. ${ }^{13,}{ }^{14}$ Colorless liquid; ${ }^{1} \mathrm{H}$ NMR $\left(200 \mathrm{MHz}, \mathrm{CDCl}_{3}+\mathrm{CCl}_{4}\right)$ : $\delta 1.30$ $1.50(\mathrm{~m}, 2 \mathrm{H}), 1.55-1.75(\mathrm{~m}, 4 \mathrm{H}), 1.90-2.08(\mathrm{~m}, 4 \mathrm{H}), 3.27(\mathrm{~s}, 4 \mathrm{H}) ;{ }^{13} \mathrm{C}$ NMR $(50.3 \mathrm{MHz}$, 
$\left.\mathrm{CDCl}_{3}+\mathrm{CCl}_{4}\right): \delta 24.84,25.94(2 \mathrm{C}), 38.15$ (2C), 42.67 (2C), 68.51; Mass (m/z): $174\left(\mathrm{M}^{+}\right)$. Anal. Calcd. for $\mathrm{C}_{8} \mathrm{H}_{14} \mathrm{~S}_{2}$ : C 55.12; $\mathrm{H}$ 8.09; S 36.79. Found C 55.20; H 8.15; S 36.85\%.

3-(1,3-Dithiolan-2-yl)-chromen-4-one. White solid; mp 113-114 ${ }^{\circ} \mathrm{C}$; IR (v max, $\left.\mathrm{CHCl}_{3}\right)$ : 1216, 1644, 2400 and $3019 \mathrm{~cm}^{-1}$; ${ }^{1} \mathrm{H}$ NMR $\left(200 \mathrm{MHz}, \mathrm{CDCl}_{3}+\mathrm{CCl}_{4}\right): \delta 3.32(\mathrm{~s}, 4 \mathrm{H}), 5.79(\mathrm{~s}, 1 \mathrm{H}), 7.35-$ $7.50(\mathrm{~m}, 2 \mathrm{H}), 7.67(\mathrm{~m}, 1 \mathrm{H}), 8.24(\mathrm{~d}, J=6 \mathrm{~Hz}, 1 \mathrm{H}), 8.32(\mathrm{~s}, 1 \mathrm{H}) ;{ }^{13} \mathrm{C} \mathrm{NMR}(50.3 \mathrm{MHz}$, $\left.\mathrm{CDCl}_{3}+\mathrm{CCl}_{4}\right): \delta 38.95(2 \mathrm{C}), 46.44,118.20,123.60,125.33,125.90,126.13,133.85,153.85$, 156.35, 176.46; Mass (m/z): $249\left(\mathrm{M}^{+}\right)$. Anal. Calcd. for $\mathrm{C}_{12} \mathrm{H}_{10} \mathrm{O}_{2} \mathrm{~S}_{2}$ : C 57.57; H 4.03; S 25.62. Found C 57.61; H 4.12; S 25.68\%.

2-(2-Methyl-1,3-dithiolan-2-yl)-furan. Liquid; IR (v max, $\mathrm{CHCl}_{3}$ ): 1150, 1498, 1645 and 2920 $\mathrm{cm}^{-1}$; ${ }^{1} \mathrm{H}$ NMR $\left(200 \mathrm{MHz}, \mathrm{CDCl}_{3}+\mathrm{CCl}_{4}\right): \delta 2.13(\mathrm{~s}, 3 \mathrm{H}), 3.45(\mathrm{~s}, 4 \mathrm{H}), 6.24-6.35(\mathrm{~m}, 2 \mathrm{H}), 7.37$ (d, $J=2 \mathrm{~Hz}, 1 \mathrm{H}) ;{ }^{13} \mathrm{C} \mathrm{NMR}\left(50.3 \mathrm{MHz}, \mathrm{CDCl}_{3}+\mathrm{CCl}_{4}\right): \delta 29.41,40.22$ (2C), 60.91, 105.90, 109.87, 141.96 (2C); Mass (m/z): $186\left(\mathrm{M}^{+}\right)$. Anal. Calcd. for $\mathrm{C}_{8} \mathrm{H}_{10} \mathrm{OS}_{2}$ : C 51.58; H 5.41; S 34.42. Found C 51.71; H 5.56; S 34.50\%.

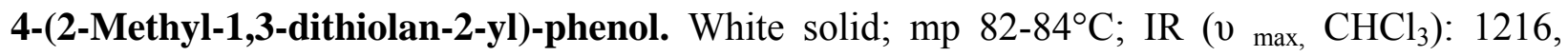
1508, 1608 and $3368 \mathrm{~cm}^{-1}$; ${ }^{1} \mathrm{H}$ NMR $\left(200 \mathrm{MHz}, \mathrm{CDCl}_{3}+\mathrm{CCl}_{4}\right): \delta 2.13(\mathrm{~s}, 3 \mathrm{H}), 3.30-3.60(\mathrm{~m}$, $4 \mathrm{H}), 5.22(\mathrm{~s}, 1 \mathrm{H}), 6.75(\mathrm{~d}, J=8 \mathrm{~Hz}, 2 \mathrm{H}), 7.60(\mathrm{~d}, J=8 \mathrm{~Hz}, 2 \mathrm{H}) ;{ }^{13} \mathrm{C} \mathrm{NMR}(50.3 \mathrm{MHz}$, $\mathrm{CDCl}_{3}+\mathrm{CCl}_{4}$ ): $\delta$ 33.71, 40.07 (2C), 68.12, 114.54 (2C), 128.10 (2C), 137.55, 154.35; Mass $(\mathrm{m} / \mathrm{z}): 212\left(\mathrm{M}^{+}\right)$. Anal. Calcd. for $\mathrm{C}_{10} \mathrm{H}_{12} \mathrm{OS}_{2}$ : C 56.57; H 5.70; S 30.20. Found C 56.74; H 5.78; S $30.29 \%$.

2-(2-Nitrophenyl)-1,3-dithiolane. ${ }^{15}$ Yellow solid; $\mathrm{mp}$ 61-62 ${ }^{\circ} \mathrm{C}$; IR (v max, $\mathrm{CHCl}_{3}$ ): 760, 1210 and $2120 \mathrm{~cm}^{-1} ;{ }^{1} \mathrm{H}$ NMR $\left(200 \mathrm{MHz}, \mathrm{CDCl}_{3}+\mathrm{CCl}_{4}\right): \delta 3.25-3.60(\mathrm{~m}, 4 \mathrm{H}), 6.20(\mathrm{~s}, 1 \mathrm{H}), 7.28-7.50$ (m, 1H), 7.55-7.67 (m, 1H), $7.87(\mathrm{dd}, J=8 \mathrm{~Hz}$ and $2 \mathrm{~Hz}, 1 \mathrm{H}), 7.90(\mathrm{dd}, J=8 \mathrm{~Hz}$ and $2 \mathrm{~Hz}, 1 \mathrm{H})$; ${ }^{13} \mathrm{C} \mathrm{NMR:} \mathrm{(50.3} \mathrm{MHz,} \mathrm{CDCl}_{3}+\mathrm{CCl}_{4}$ ): $\delta 39.79$ (2C), 50.47, 124.36 (2C), 128.31, 130.30, 133.02, 136.94, 148.34; Mass (m/z): $227\left(\mathrm{M}^{+}\right)$. Anal. Calcd. for $\mathrm{C}_{9} \mathrm{H}_{9} \mathrm{NO}_{2} \mathrm{~S}_{2}: \mathrm{C} 47.56 ; \mathrm{H} 3.99 ; \mathrm{N}$ 6.16; S 28.21. Found C 47.68; H 4.12; N 6.25; S 28.30\%.

5-(Bis-ethylsulfanyl-methyl)-1,2,3-trimethoxybenzene. To a mixture of 3,4,5-trimethoxy benzaldehyde $(0.50 \mathrm{~g}, 2.55 \mathrm{mmol})$ and ethanethiol $(0.33 \mathrm{~g}, 5.35 \mathrm{mmol})$ in water $(5 \mathrm{ml})$ was added aqueous hydrobromic acid $(48 \% \mathrm{w} / \mathrm{w}, 0.05 \mathrm{ml}, 0.41 \mathrm{mmol})$. The reaction mixture was stirred at room temperature for $30 \mathrm{~min}$ (reaction was monitored by thin layer chromatography). It was then extracted with chloroform $(2 \times 15 \mathrm{ml})$ and the combined organic layer was washed with $10 \%$ sodium hydroxide solution $(10 \mathrm{ml})$ followed by water $(10 \mathrm{ml})$ and brine $(5 \mathrm{ml})$. Concentration of organic layer under reduced pressure and purification of residue by passing through silica gel furnished 5-(bis-ethylsulfanyl-methyl)-1,2,3-trimethoxybenzene (0.71 g, 92 \%) as a colorless thick liquid.

${ }^{1} \mathrm{H}$ NMR (200 MHz, $\left.\mathrm{CDCl}_{3}+\mathrm{CCl}_{4}\right): \delta 1.22(\mathrm{t}, J=7 \mathrm{~Hz}, 6 \mathrm{H}), 2.57(\mathrm{~m}, 4 \mathrm{H}), 3.30-3.81(\mathrm{~s}, 3 \mathrm{H}), 3.85$ $(\mathrm{s}, 6 \mathrm{H}), 4.82(\mathrm{~s}, 1 \mathrm{H}), 6.66(\mathrm{~s}, 2 \mathrm{H}) ;{ }^{13} \mathrm{C} \mathrm{NMR}\left(50.3 \mathrm{MHz}, \mathrm{CDCl}_{3}+\mathrm{CCl}_{4}\right): \delta 13.63(2 \mathrm{C}), 25.61$ (2C), 52.34, 55.39 (2C), 59.83, 104.31 (2C), 135.41, 136.99, 152.47 (2C); Mass (m/z): $302\left(\mathrm{M}^{+}\right)$. Anal. Calcd. for $\mathrm{C}_{14} \mathrm{H}_{22} \mathrm{O}_{3} \mathrm{~S}_{2}$ : C 55.60; H 7.33; S 21.20. Found C 55.74; H 7.45; S 21.27\%. 


\section{Acknowledgements}

P.D.S. thanks CSIR, New Delhi for the award of Senior Research Fellowship.

\section{References}

1. (a) Corey, E. J.; Seebach, D. Angew. Chem., Int. Ed. 1965, 4, 1075. (b) Corey, E. J.; Seebach, D. J. Org. Chem. 1975, 40, 231. (c) Seebach, D. Angew. Chem., Int. Ed. 1969, 8, 639 (d) Grobel, B. T.; Seebach, D. Synthesis 1977, 357. (d) Bulman Page, P. C.; Van Niel, M. B.; Prodger, J. C. Tetrahedron 1989, 45, 7643.

2. (a) Greene, T. W.; Wuts, P. G. M. Protective Groups in Organic Synthesis $3^{\text {rd }}$ Edn., Wiley : New York, 1999, 329. (b) Corey, E. J.; Erickson. B. W. J. Org. Chem. 1971, 36, 3553. (c) Garlaschelli, L.; Vidari, G. Tetrahedron Lett. 1990, 31, 5815. (d) Ong, B. S. Tetrahedron Lett. 1980, 4225. (e) Kumar, V.; Dev, S. Tetrahedron Lett. 1983, 24, 1289. (f) Ravindranathan, T.; Chavan, S. P.; Tejwani, R. B.; Varghese, J. P. J. Chem. Soc., Chem. Commun. 1991, 24, 1750. (g) Funabashi, M.; Arai, S.; Shinohara, M. J. Carbohydr. Chem. 1999, 18, 333. (h) Tietze, L. F.; Weigand, B.; Wulff, C. Synthesis 2000, 69. (i)Yadav, G. D.; Nair, J. J. Microporous Mesoporous Mater. 1999, 33, 1. (j) Battaglia, L.; Pinna, F.; Strukul, G. Can. J. Chem. 2001, 79, 621. (k) Samajdar, S; Basu, M. K.; Becker, F. F.; Banik, B. K. Tetrahedron Lett. 2001, 42, 4425. (1) Firouzabadi, H.; Iranpoor, N.; Amani, K. Synthesis 2002, 59. (m) Ranu, B. C.; Das, A.; Samanta, S. J. Chem. Soc., Perkin Trans. 1 2002, 13, 1520. (n) Ahmed, K.; Gagan, C. Tetrahedron Lett. 2002, 43, 1347. (o) Yadav, J. S.; Reddy, B. V. S.; Pandey, S. K. Synth. Commun. 2002, 32, 715.

3. Lubineau, A.; Auge, J. Topics in Current Chemistry 1999, 206, 1.

4. Ceschi, M. A.; Fleix, L. A.; Peppe, C. Tetrahedron Lett. 2000, 41, 9695

5. Manabe, K.; Iimura, S.; Sun, X. M.; Kobayashi, S. J. Am. Chem. Soc. 2002, 124, 11971.

6. (a) Gajare, A. S.; Shingare, M. S.; Bandgar, B. P. J. Chem. Research (S). 1998, 452. (b) Firouzabadi, H.; Iranpoor, N.; Amani K. Synthesis 2002, 59. (c) Ranu, B. C.; Das, A.; Samanta, S. Synlett 2002, 5, 727. (d) Firouzabadi, H.; Iranpoor, N.; Hazarkhani, H. J. Org. Chem. 2001, 66, 7527.

7. Jnaneshwara, G. K.; Barhate, N. B.; Sudalai, S.; Deshpande, V. H.; Wakharkar, R. D; Gajare, A. S.; Shingare, M. S.; Sukumar, R. J. Chem. Soc., Perkin Trans. 1 1998, 1, 965.

8. Diez, E.; Lopez, A. M.; Pareja, C.; Martin, E.; Fernandez, R.; Lassaletta, M. J. Tetrahedron Lett. 1998, 39, 7955.

9. Vogel's Textbook of Practical Organic Chemistry, $5^{\text {th }}$ Edn, Longman: Harlow, 1989.

10. Sankararaman, S.; Saraswathy, V. G. J. Org. Chem. 1994, 59, 4665.

11. Perni, R. B. Synth. Commun. 2001, 31, 1205.

12. Kamitori, T.; Hojo, K.; Masud, R.; Kimura, T.; Yoshida, T. J. Org. Chem. 1986, 51, 1427.

13. Deka, N.; Sarma, J. C. Chem. Lett. 2001, 749.

14. Patney, H. K. Tetrahedron Lett. 1991, 32, 413.

15. Kamal, A.; Chouhan, G.; Ahemd, K. Tetrahedron Lett. 2002, 43, 6947. 